\title{
Tracking and Vertexing for the Heavy Photon Search Experiment
}

\author{
Timothy K. Nelson ${ }^{* \dagger}$ \\ SLAC National Accelerator Laboratory \\ E-mail: tknelsoneslac.stanford.edu
}

\begin{abstract}
Recent astrophysical data motivates searches for a massive, dark sector analog to the photon. The preferred parameter space for this "heavy photon" requires very high luminosity for direct production, favoring fixed target experiments, and results in long-lived decays of these new force carriers to pairs of light, charged fermions. Identification of these states on large backgrounds requires precision tracking and vertexing at high rates in a difficult experimental environment. The Heavy Photon Search, or HPS, proposes a novel silicon tracking and vertexing detector, the HPS Silicon Vertex Tracker (SVT), to search for these states in a fixed target experiment at JLab. The HPS SVT employs actively cooled silicon microstrip sensors with fast readout electronics directly downstream of a target and inside of a dipole magnet to instrument a large acceptance with a relatively small detector. As a first step; a simple, small scale version of this detector has been assembled and operated on beam at JLab.
\end{abstract}

The 21st International Workshop on Vertex Detectors

16-21 September 2012

Jeju, Korea

\footnotetext{
* Speaker.

†n behalf of the HPS Collaboration.
} 


\section{The Physics of the HPS Experiment}

One of the more striking anomalies in recent astrophysical observations is an excess of high energy electrons and positrons consistent with a large, unknown source of pair production [1][2]. The existence of a massive, hidden sector vector boson; a "dark photon;" that mixes kinetically with the Standard Model photon and mediates annihilation or decay of dark matter particles is a theoretically attractive explanation of this cosmic-ray excess [3][4].

There are two necessary parameters that determine the properties of this "heavy photon," designated the $\mathrm{A}^{\prime}$. The first is the mixing of this state with the Standard Model photon, $\varepsilon$, which results in a coupling of the $\mathrm{A}^{\prime}$ to Standard Model fermions with a strength of $\varepsilon e$. In simple models with kinetic mixing, $\varepsilon$ is favored to be in the range of $10^{-2}-10^{-5}$. The second is the mass of the $\mathrm{A}^{\prime}$. Under the assumption that this mass is generated via a Higgs-like symmetry breaking, the mass would be roughly of the order $\sqrt{\varepsilon} m_{W} \sim \mathrm{MeV}-\mathrm{GeV}$. These ranges are further motivated by explanations of other dark matter anomalies involving non-minimal dark sectors [5] and the fact that no similar excess in proton-antiproton pairs is observed, which would be simply explained if $m_{\mathrm{A}^{\prime}}<2 m_{p}[6]$.

Searches for these heavy photons with high-intensity, fixed target experiments offer the best sensitivity in this region of the parameter space [7]. In these experiments, an $\mathrm{A}^{\prime}$ is radiated off the incident electron, much like a bremsstrahlung photon, which subsequently decays to a pair of Standard Model fermions. However, achieving sensitivity to this parameter space presents a number of challenges. At larger couplings, large data samples are required to identify a small invariant mass peak on significant background. At smaller couplings, the $\mathrm{A}^{\prime}$ becomes long-lived, which enables sensitivity from extremely high-luminosity beam-dump experiments that screen out the Standard Model backgrounds. However, in the intermediate region, lifetimes are too short to screen out backgrounds with a shield, but signal cross sections are so small that collecting large enough event samples to distinguish the signal requires unacceptable data rates or running times. Gaining sensitivity to this region requires a fast detector capable of precision mass reconstruction of $\mathrm{A}^{\prime}$ final states together with the best possible rejection of prompt vertexes to eliminate backgrounds. The HPS experiment aims to achieve these goals by placing a compact silicon and vertexing detector $(\approx 1 \mathrm{~m}$ long $)$ immediately downstream $(\approx 10 \mathrm{~cm})$ of a thin target $\left(\approx 0.25 \mathrm{X}_{0}\right.$ tungsten $)$ and inside of a dipole magnet $(\approx 1 \mathrm{~T})$ [8].

\section{The HPS detector}

The HPS experiment is proposed to run in Hall B at JLab using the CEBAF electron beam. This electron beam is delivered at $499 \mathrm{MHz}$ at energies ranging from $1.1 \mathrm{GeV}$ to $5.5 \mathrm{GeV}$ and currents up to $800 \mathrm{nA}$. An energy upgrade to be completed in 2014 doubles this range. The kinematics of $\mathrm{A}^{\prime}$ production typically results in final state particles within a few degrees of the incoming

beam, especially at low $m_{\mathrm{A}^{\prime}}$. Because of this, the apparatus must accommodate passage of the beam downstream of the target and operate as close to that beam as possible. Because background rates in this region from the scattered beam are very large, a fast trigger and excellent time tagging of hits are required to select interesting data and reduce the bandwidth required to transfer data from the detector. This method of background reduction is the motivation for operating HPS in a 
nearly continuous beam: in a beam with large per-bunch charge, background from a single bunch would fully occupy the detector at the required beam intensity. At masses below $2 m_{\mu}$ only decays to electrons are allowed, so the key trigger comes from a lead-tungstate calorimeter with $250 \mathrm{MHz}$ FADC readout. Above the di-muon threshold, a downstream muon detector completes the trigger acceptance and may allow triggering for pion final states required for sensitivity near the $\rho$ mass.

\section{The HPS SVT}

The design of the SVT presents a number of significant challenges. First, the $\mathrm{A}^{\prime}$ decay products have momenta in the range of $1 \mathrm{GeV}$, so multiple scattering dominates mass and vertexing errors for any possible material budget. Therefore, the construction of the SVT must place the smallest amount of material in the tracking volume. Second, the signal yield for long-lived $\mathrm{A}^{\prime}$ will be very small, so the rejection of prompt vertexes must be exceedingly pure, on the order of $10^{-7}$, in order to eliminate all prompt backgrounds. This requires great care in design, calibration, and operation of the detector. Finally, the passage of degraded primary beam through the apparatus creates a region of extreme occupancy and radiation that is critical for sensitivity to low-mass $\mathrm{A}^{\prime}$ that have decay products nearly collinear with the beam. This places low-mass acceptance in opposition to tracking and vertexing purity and the longevity of the detector.

It is this last challenge that has the most interesting and obvious impacts on the design of the SVT. To achieve good vertexing performance, the first layer must be placed no more than about $10 \mathrm{~cm}$ downstream of the target. At that distance, it is found that the active region of a sensor can be placed as close to $1.5 \mathrm{~mm}$ from the center of the beam, defining a $15 \mathrm{mrad}$ "dead zone" in the detector around the plane of the primary beam. At the edge of this "dead zone," the radiation dose approaches $10^{15}$ electrons $/ \mathrm{cm}^{2} /$ month, or roughly $3 \times 10^{13} 1 \mathrm{MeV}$ neutron equivalent $/ \mathrm{cm}^{2} / \mathrm{month}$, as shown in Figure 1(a) [9]. This requires the sensors to be actively cooled. Meanwhile, very

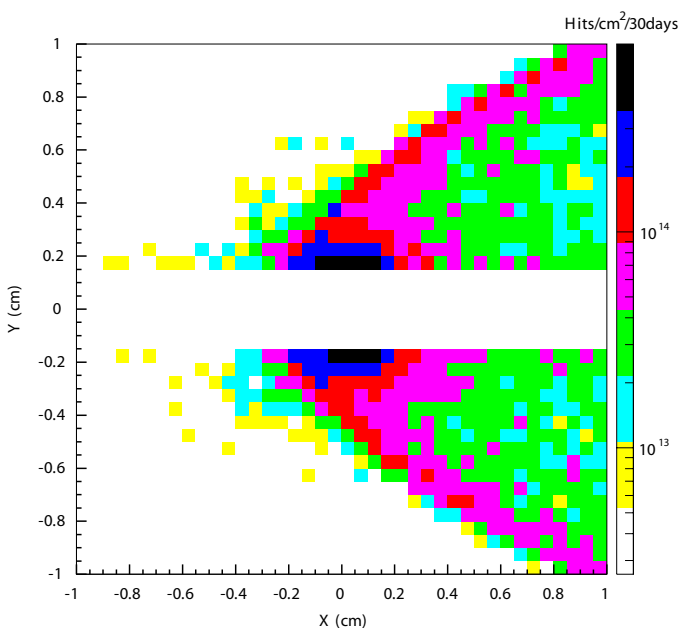

(a) Electron fluences: electrons bend towards $+x$.

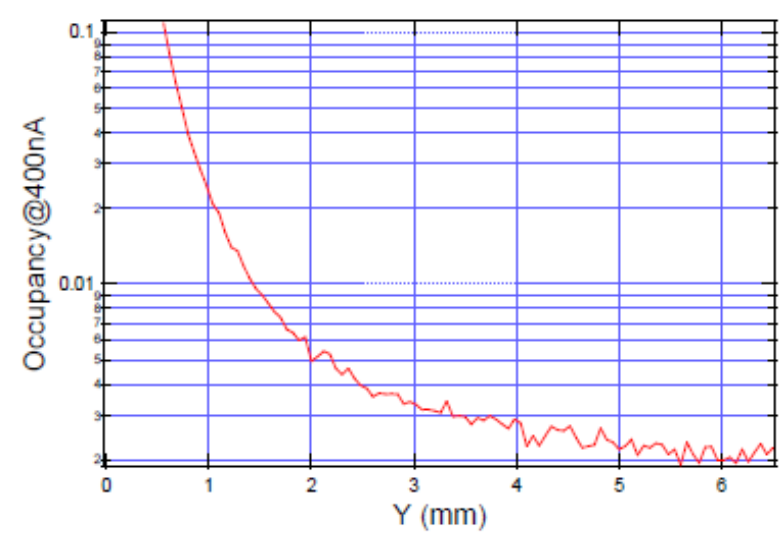

(b) The occupancy in an $8 \mathrm{~ns}$ window in 60 micron strips.

Figure 1: The environment near the dead zone $10 \mathrm{~cm}$ from the target.

low-energy delta rays from beam gas interactions multiply the density of background hits, so the 
SVT must operate inside the beam vacuum. Finally, in order protect the sensors, the detector must be movable so that it can be retracted during periods of uncertain beam conditions.

The sensors for the SVT are selected according to a number of requirements. First, the sensors must be radiation tolerant to approximately $1.5 \times 10^{14} 1 \mathrm{MeV}$ neq $/ \mathrm{cm}^{2}$ for a six month run. This corresponds to about $4 \mathrm{MHz} / \mathrm{mm}^{2}$, so the sensor and readout technology must be capable of handling very high local occupancies. Since sensitivity is limited by multiple scattering, a material budget of less than $1 \% X_{0} /$ layer is imperative and far less is desirable. The best vertex resolution with a small beam spot $(\approx 10 \mu \mathrm{m})$ requires single-hit resolution better than $20 \mu \mathrm{m}$ in both measurement coordinates. Finally, the sensor technology must be mature and readily available at low cost. Surprisingly, we find that silicon microstrips are the best match to these requirements, and a supply of appropriate microstrip sensors purchased from the Hamamatsu Photonics Corporation for the Run $2 \mathrm{~b}$ upgrade of $\mathrm{D} \emptyset[10]$ are readily available. These are $p+$ on $n$, single sided, AC coupled, polysilicon-biased sensors fabricated on $<100>$ silicon and have 30(60) micron sense(readout) pitch over their $4 \mathrm{~cm} \times 10 \mathrm{~cm}$ surface. Although a maximum bias of $350 \mathrm{~V}$ is specified, the vast majority are operable to $1000 \mathrm{~V}$ and therefore remain fully depleted to a dose of approximately $1.5 \times 10^{14} 1 \mathrm{MeV}$ neq $/ \mathrm{cm}^{2}$.

Because the regions of high occupancy are small spots in two dimensions, only a short length of any one strip sees significant occupancy, and the strips in that region act as long pixels. However, the rates are still very high and lowering the peak occupancy in the sensors to approximately $1 \%$ for tracking requires a trigger window and tagging of hit times to roughly $8 \mathrm{~ns}$, as shown in Figure 1(b). The FADC readout for the ECal and muon system are capable of this, but achieving achieving $2 \sigma$ efficiency for silicon hits then requires $2 \mathrm{~ns}$ time resolution for the hits in the SVT. This performance can be achieved with the APV25 readout ASIC developed for the CMS experiment at CERN [11]. When operated in "multi-peak mode", the APV25 captures successive samples of the output of the shaper in sets of three. By fitting the known $C R-R C$ shaping curve to these samples, the initial time of the hit can be determined to a precision of $2 \mathrm{~ns}$ for $\mathrm{S} / \mathrm{N}>25$, an achievable figure with our sensors if read out individually [12]. For the SVT, six-sample readout and the shortest possible shaping time ( $35 \mathrm{~ns}$ ) will be used to best distinguish hits that overlap in time.

A realistic simulation of the detector using the java-based org.lcsim package and including full backgrounds has been used to optimize the layout for acceptance, tracking efficiency, mass resolution and prompt vertex rejection [13]. The layout of the HPS SVT is summarized in Table 1 and shown in Figure 2. There are six measurement stations, or "layers," placed immediately downstream of the target. Each layer comprises a pair of closely-spaced planes and each plane is responsible for measuring a single coordinate, or "view". Introduction of a stereo angle between the two planes of each layer enables three-dimensional tracking and vertexing. The requirements of the tracking system are discernibly reflected in the layout. The 90-degree stereo of the first three layers provides a pair of high-resolution 3-d space points for vertexing. The small-angle stereo of the last three layers minimize ghost hits to improve pattern recognition while still providing sufficient pointing resolution into Layer 3 for robust hit association in the dense environment there. Meanwhile, the presence of a precise bend-plane measurement in every single layer assures the best possible momentum resolution.

The APV25 chips are hosted on simple FR4 hybrids since these hybrids and their cooling system are outside the tracking volume. Along with the sensors, these are assembled into large 


\begin{tabular}{lcccccc}
\hline \hline Layer & 1 & 2 & 3 & 4 & 5 & 6 \\
\hline nominal $z$, from target $(\mathrm{cm})$ & 10 & 20 & 30 & 50 & 70 & 90 \\
Stereo Angle & $90^{\circ}$ & $90^{\circ}$ & $90^{\circ}$ & $50 \mathrm{mrad}$ & $50 \mathrm{mrad}$ & $50 \mathrm{mrad}$ \\
Bend plane resolution $(\mu \mathrm{m})$ & $\approx 6$ & $\approx 6$ & $\approx 6$ & $\approx 6$ & $\approx 6$ & $\approx 6$ \\
Non-bend plane Resolution $(\mu \mathrm{m})$ & $\approx 6$ & $\approx 6$ & $\approx 6$ & $\approx 120$ & $\approx 120$ & $\approx 120$ \\
\# bend-plane sensors & 4 & 4 & 6 & 10 & 14 & 18 \\
\# non-bend-plane sensors & 2 & 2 & 4 & 10 & 14 & 18 \\
Nominal dead zone $(\mathrm{mm})$ & \pm 1.5 & \pm 3.0 & \pm 4.5 & \pm 7.5 & \pm 10.5 & \pm 13.5 \\
Power consumption $(\mathrm{W})$ & 10.4 & 10.4 & 17.3 & 35 & 48 & 62 \\
\hline \hline
\end{tabular}

Table 1: The proposed layout of the HPS SVT.

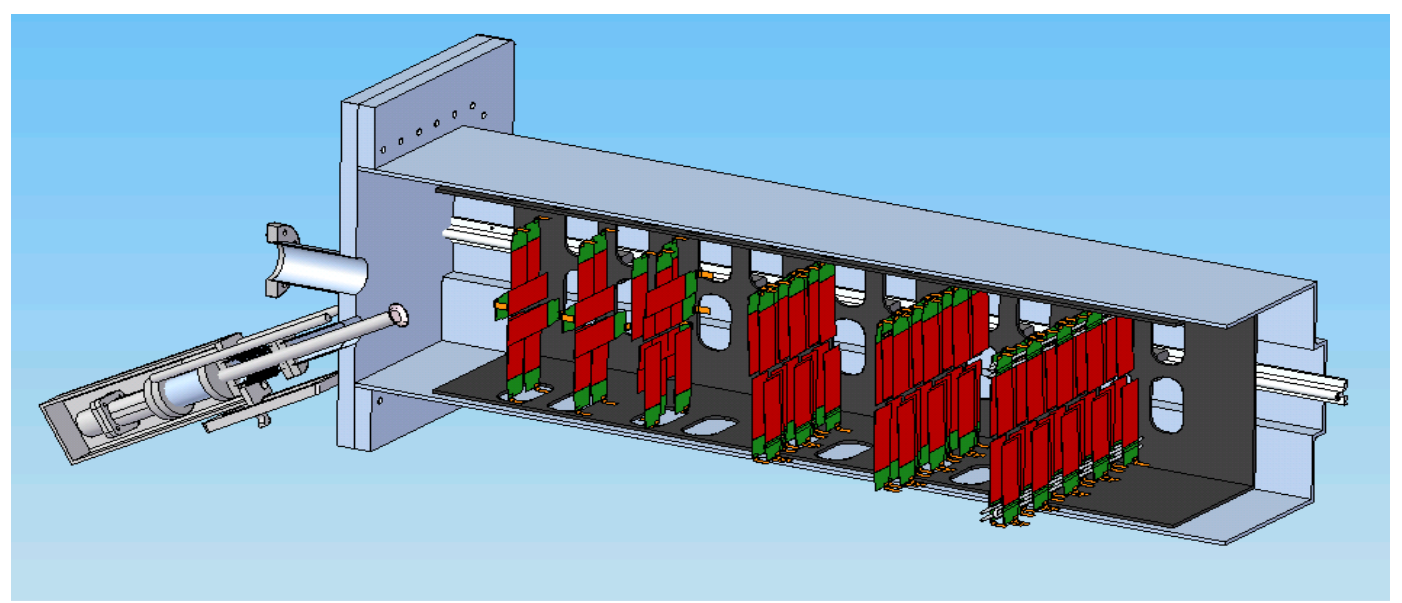

Figure 2: The conceptual design of the SVT showing the six double-sided layers of silicon inside the vacuum chamber. The target foil, shown at left, rotates to prevent damage from overheating.

multi-sensor modules, one for each tracking view. The module support structures help conduct heat along the sensors to integrated cooling located under the hybrids. Since heating from leakage current is only significant at a single small spot on the sensor, the dominant heat load, even after irradiation, is radiant heat from the inside wall of the vacuum chamber, less than $0.5 \mathrm{~W}$ per sensor. A blanket of multilayer insulation will reduce this heat load and the temperature drop along the sensor to result in the lowest possible operational temperature for the silicon. The modules comprising the top and bottom halves of the tracker will be mounted in a support structure that slides into and out of the vacuum chamber on rails. A motion control system will allow the vertical position of tracker planes to be modified remotely in response to the experimental environment.

After stringent track quality and isolation requirements, the tracking efficiency over the full momentum range exceeds $95 \%$ and purity is excellent with all hits correctly assigned in $99 \%$ of tracks. The vertex-constrained mass resolution is approximately $1 \%$ and impact parameter error approaches $30 \mu \mathrm{m}$ at high momentum. Due to the far-forward nature of the decay products, this results in decay length resolution on the order of $1 \mathrm{~mm}$ with a strong dependence on the $\mathrm{A}^{\prime}$ mass. 
However, the critical vertexing task is the rejection of prompt vertexes, where the measure is the decay length cut that results in $10^{-7}$ rejection of prompt vertexes. As shown in Figure 3(a), the distribution of background vertexes has short tails after full selections, enabling the sensitivity required for the experiment, shown in Figure 3(b).

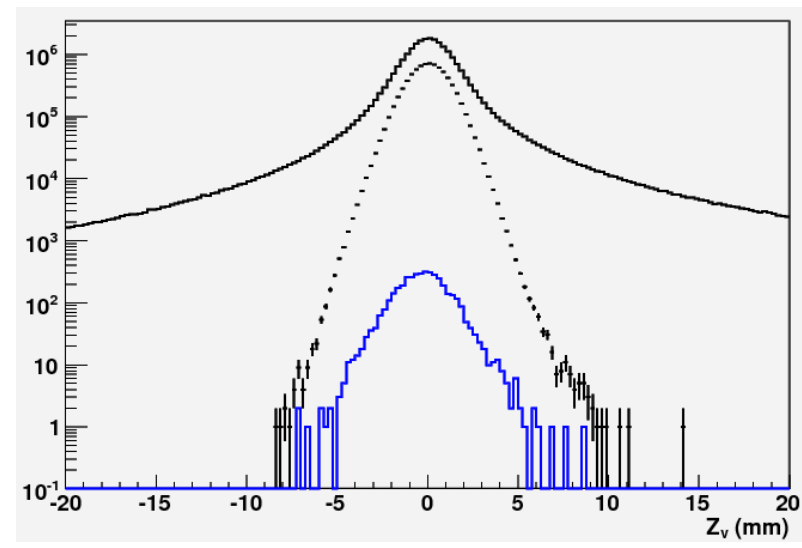

(a) The decay length from all track pairs (black histogram), track pairs after selections (black points) and selected pairs with at least one incorrectly assigned hit (blue histogram.)

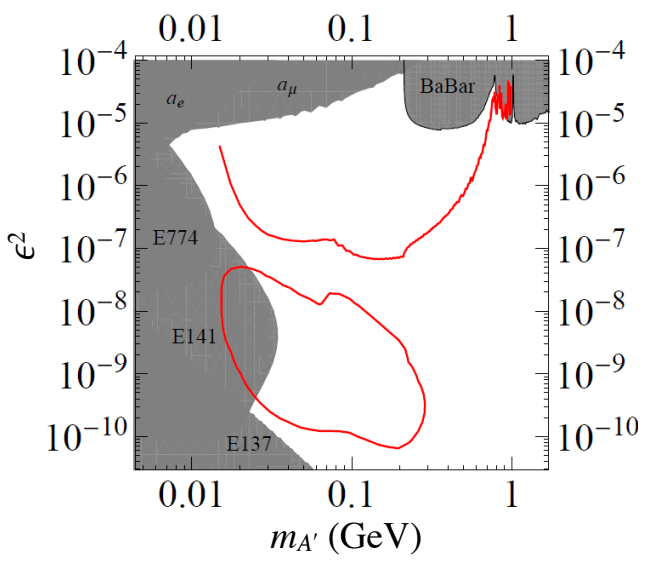

(b) The reach of HPS for a pair of three month runs at beam energies of $2.2 \mathrm{GeV}$ and $6.6 \mathrm{GeV}$. Contours are two-sigma exclusions for a null result.

Figure 3: The vertex resolution and resulting reach of the HPS experiment.

\section{The HPS Test Run}

The HPS Experiment was approved by the JLab PAC in early 2011 contingent upon a successful "Test Run" to prove the feasibility of the concept. With CEBAF operation ending in May 2012 for the energy upgrade, this left little time to design, develop, assemble, test, install, commission, and run this experiment to avoid waiting until 2014. Fortunately, two key elements were already available. First, there is an magnet chicane in Hall $\mathrm{B}$ with a large analyzing magnet and the bore of this magnet contains a vacuum chamber large enough to build a small tracker. Second, an existing $\mathrm{PbWO}_{4}$ calorimeter could be reconfigured as an electron trigger, instrumented with new FADC readout under development for another experiment.

The layout of the HPS Test SVT is shown in Figure 4 and summarized in Table 2. The vacuum chamber is too small vertically to accommodate the 90-degree stereo layers planned for the full experiment to optimize the vertex resolution. Instead, 100 milliradian stereo is used in the first three layers to provide higher-resolution 3-d space points for vertexing. The 50 milliradian stereo of the last two layers breaks the tracking degeneracy of having five identical layers and minimizes fakes from ghost hits, improving pattern recognition while still providing sufficient pointing resolution into Layer 3 for robust hit association in the denser environment there. Altogether, this layout comprises 20 sensors and hybrids and 100 APV25 chips for a total of 12780 readout channels. The Test SVT is built in two separate halves that are mirror reflections of one another about the plane of the nominal electron beam. Each half consists of five, double-sided modules mounted on a support plate that provides services to the modules and allows them to be moved as a group relative 


\begin{tabular}{lccccc}
\hline \hline Layer & 1 & 2 & 3 & 4 & 5 \\
\hline nominal $z$, from target $(\mathrm{cm})$ & 10 & 20 & 30 & 50 & 70 \\
Stereo Angle $(\mathrm{mrad})$ & 100 & 100 & 100 & 50 & 50 \\
Bend plane resolution $(\mu \mathrm{m})$ & $\approx 60$ & $\approx 60$ & $\approx 60$ & $\approx 120$ & $\approx 120$ \\
Non-bend plane Resolution $(\mu \mathrm{m})$ & $\approx 6$ & $\approx 6$ & $\approx 6$ & $\approx 6$ & $\approx 6$ \\
\# sensors & 4 & 4 & 4 & 4 & 4 \\
Nominal dead zone $(\mathrm{mm})$ & \pm 1.5 & \pm 3.0 & \pm 4.5 & \pm 7.5 & \pm 10.5 \\
Power consumption $(\mathrm{W})$ & 6.9 & 6.9 & 6.9 & 6.9 & 6.9 \\
\hline \hline
\end{tabular}

Table 2: Layout of the HPS Test SVT. Note the elimination of one layer and 90-degree stereo, and the use of only small, single-sensor-sized double-sided modules both above and below he dead zone.

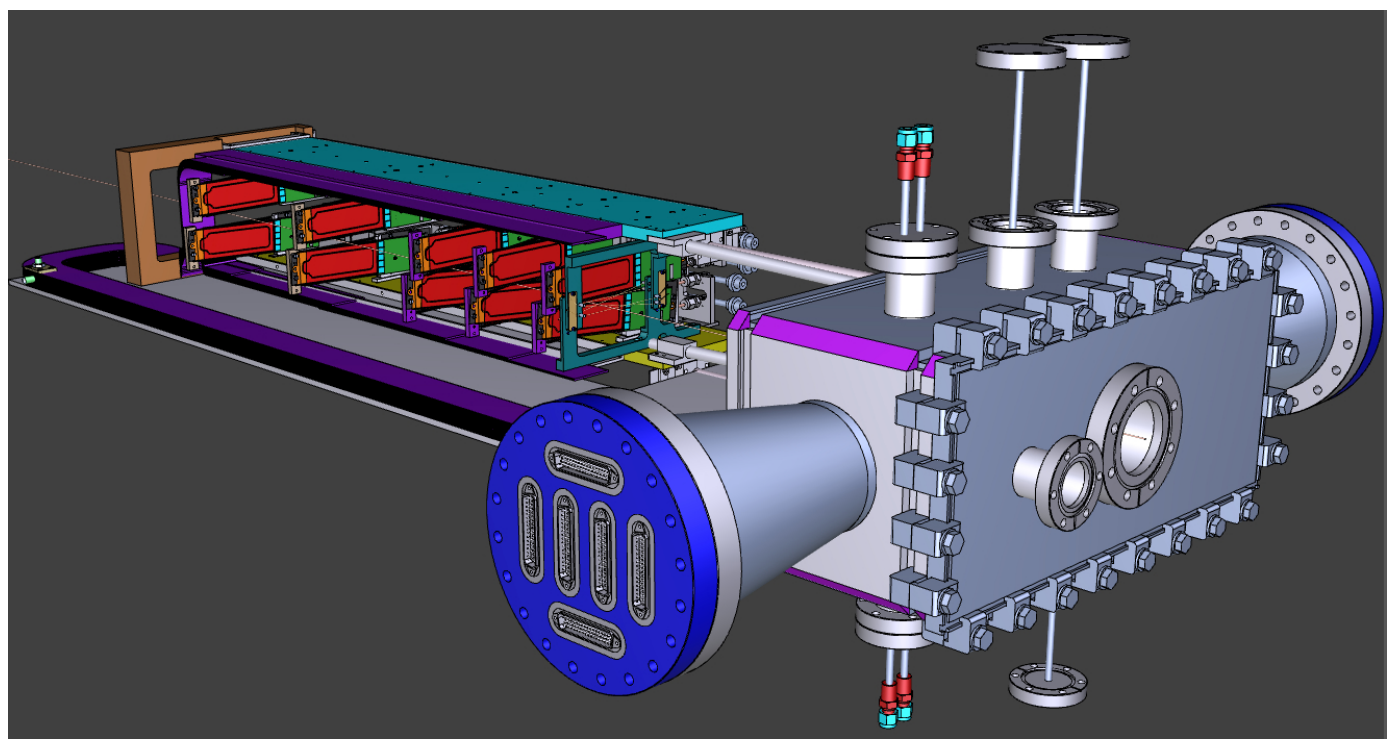

Figure 4: A rendering of the Test SVT showing the modules on their support plates held by the hinged $\mathrm{C}$-support on the left and the motion levers on the right. The sensors are shown in red and the hybrids in green. The beam enters from the right through a vacuum box with flanges for services.

to the dead zone. The two halves of the tracker are connected to hinges mounted on a C-shaped support just beyond layer 5 that defines the nominal spacing between the upper and lower halves of the tracker. A shaft attached to each support plate in front of layer 1 extends upstream and connects to a linear shift that transfers motion into the vacuum box through bellows to open and close the tracker around the dead zone. The $\mathrm{C}$-support is mounted to an aluminum baseplate that defines the position of the SVT with respect to the vacuum chamber. Figure 5 shows a photograph of both completed detector halves prior to final assembly.

The sensor modules for the SVT consist of a pair of identical half-modules, sandwiched backto-back around an aluminum cooling block at one end and a similar PEEK spacer block at the other. Figure 6 shows a prototype module assembly. The cooling block provides the primary mechanical support for the module as well as cooling via copper tubes pressed into grooves in the plates. The spacer block defines the spacing between the sensors at the far end of the module, stiffens the 


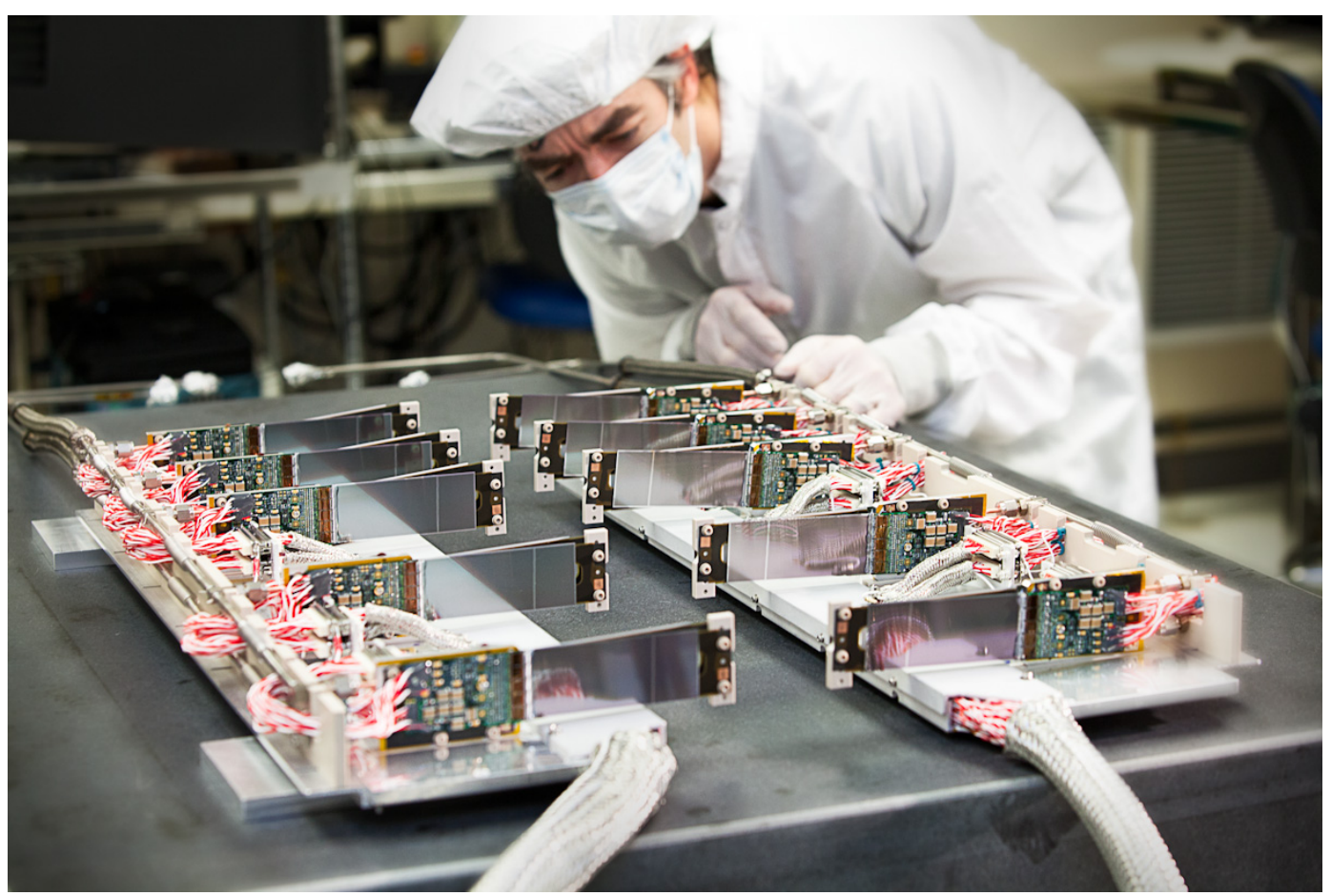

Figure 5: Both halves of the HPS Test SVT being inspected by the author after final assembly at SLAC. The cooling manifolds and integrated cable runs are clearly seen.

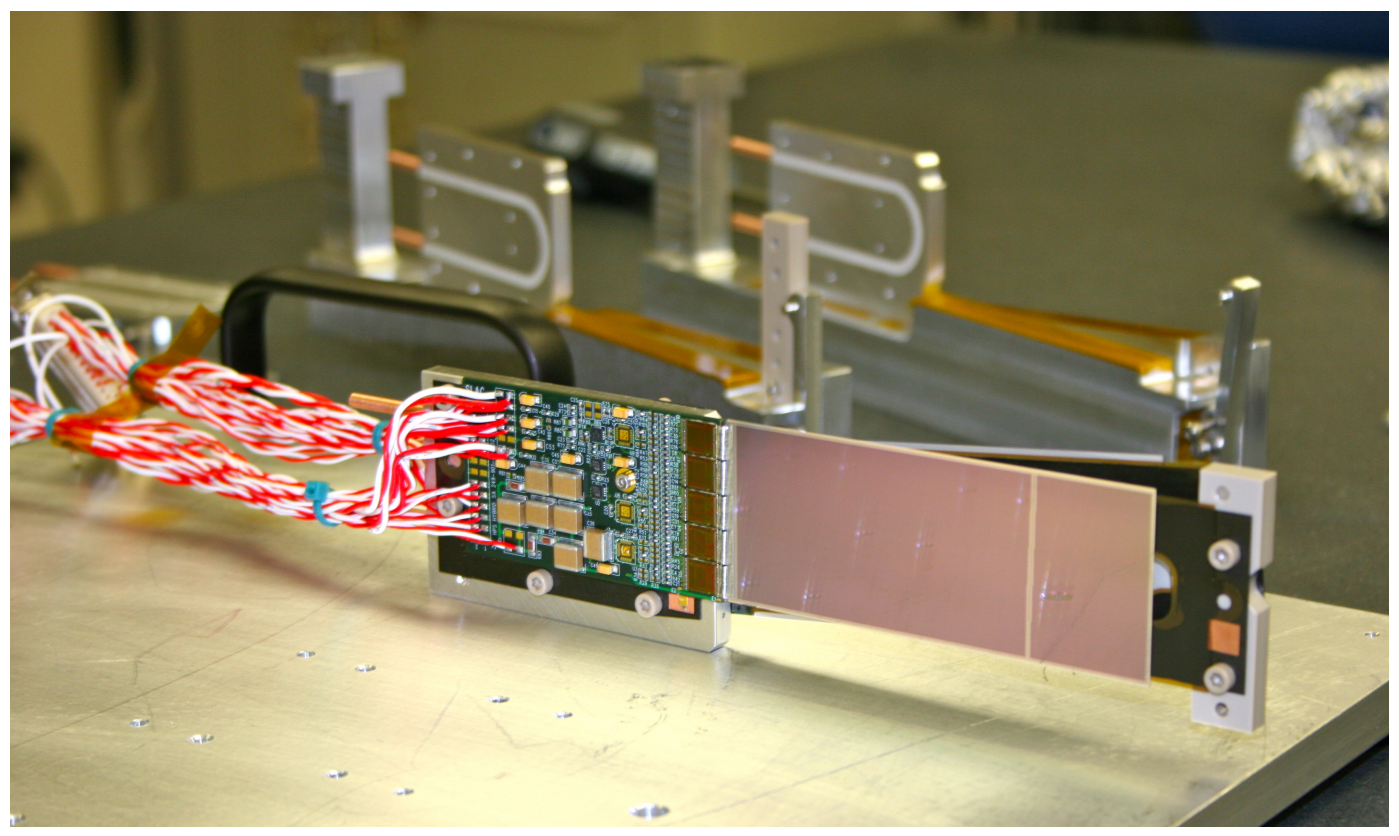

Figure 6: A prototype module assembly (foreground) with the $50 \mathrm{mrad}$ (left) and $100 \mathrm{mrad}$ (right) module assembly fixtures in the background. A pair of cooling blocks and a spacer block can be seen on the fixtures. 
module structure, and improves the stability of the sensor alignments.

Each half module consists of a single sensor and a hybrid electronic readout board glued to a polyamide-laminated carbon fiber composite backing. A window is machined in the carbon fiber leaving only a frame around the periphery of the silicon to minimize material. A $50 \mu \mathrm{m}$ sheet of polyamide is laminated to the surface of the carbon fiber with $1 \mathrm{~mm}$ overhang at all openings to ensure good isolation between the backside of the sensor, carrying high-voltage bias, and the carbon fiber which is held near ground. The average support material in the tracking volume is approximately $0.06 \% X_{0}$ per double-sided module for a total of $0.7 \%$ per layer.

The SVT DAQ is a SLAC-standard system using consisting of a single ATCA crate with two Cluster On Board (COB) cards and a $10 \mathrm{~Gb} / \mathrm{s}$ switch [14]. The COBs connect to Rear Transition Modules (RTM) housing 14-bit ADCs the accept input data from the APV25. The COB house FPGA-based Data Processing Modules (DPM) and the Trigger Interface (TI) to the ECal trigger. This system is capable of $20 \mathrm{KHz}$ operation, whereas the goal for the full experiment is $50 \mathrm{kHz}$. Power is provided to each hybrid using CAEN power supplies from the decommissioned CDF SVXII detector.

Hybrids and half-modules were assembled at SLAC. Mounting APV25 chips and all wirebonding and QA testing were performed at UCSC. Of the sensors tested during production, 90\% were capable of $1000 \mathrm{~V}$ bias. Hybrids underwent quick QA testing and each half-module was run at low temperature $\left(\approx 5^{\circ} \mathrm{C}\right)$ and fully characterized for pedestals, gains, noise and time response after assembly. All 29 production modules were assembled between mid-February and the end of March, 2012. Module yields were excellent for such a small production. Of 165 APV chips acquired, 150 were used to assemble 30 production hybrids, of which 29 passed QA testing. Of 29 modules built, 28 passed QA testing, leaving 8 spare modules after completion of the HPS Test SVT. Full-module assembly and mechanical surveys were performed at SLAC in early April 2012 before final assembly, testing and shipping of the SVT to JLab on April 11. At JLab, the entire detector was integrated with the full DAQ and the power supplies for the first time in the cleanroom, before moving the module-loaded support plates to Hall B for final mechanical assembly and installation inside of the vacuum chamber on April 19.

The experimental schedule in Hall B did not permit running with electron beam. Instead, HPS Test ran parasitically with a photon beam, tracking conversions from an upstream target. Despite intermittent synchronization problems with a small number of APV25 chips, the Test SVT performed well and roughly two weeks into commissioning began successfully reconstructing tracks. Calibration and analysis of the data continues but some key results establishing the feasibility of the experiment, tracking efficiency and hit time resolution, are shown in Figures 7(a) and 7(b) .

\section{Status and Plans}

In the process of developing the HPS Test design, it was found that this simple system was capable of delivering a surprising fraction of the physics potential anticipated for the full experiment. With this in mind, we are planning to propose a new design for the HPS SVT that builds upon the HPS Test SVT, principally by addressing the compromises made for HPS Test to ensure the best possible performance for $\mathrm{A}^{\prime}$ physics within the envelope of the existing beam line layout and analyzing magnet. This design uses the same sensors, readout chips, and module concept retaining 


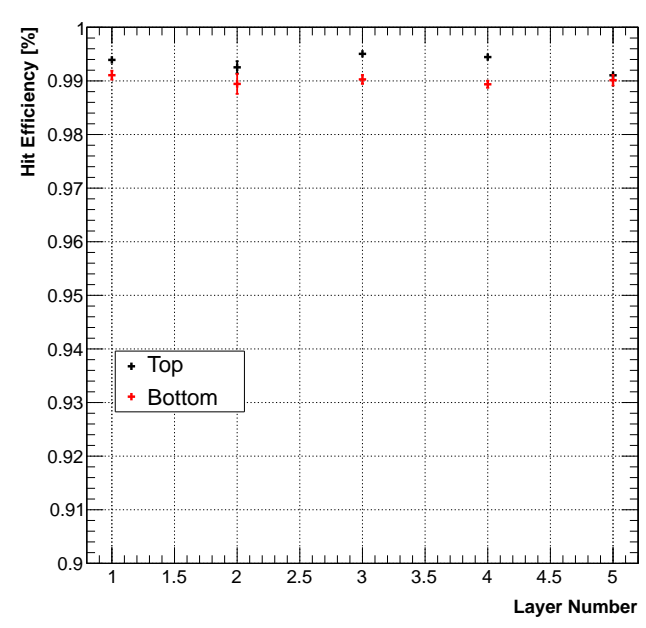

(a) The efficiency for placing hits on tracks for each layer of the Test SVT.

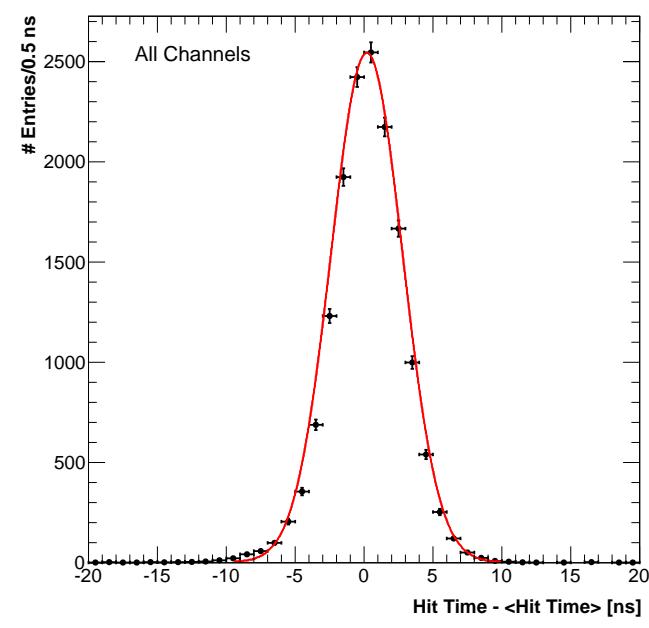

(b) The reconstructed time of individual hits on a track relative to the mean hit time for the track.

Figure 7: Early SVT performance results from the HPS Test Run.

the most successful elements of the HPS Test SVT and addressing the weaknesses identified during assembly and operation to ensure the success of the experiment. The new layout restores the sixth layer for more robust tracking, adds acceptance in the deeper layers to increase sensitivity and provides better silicon cooling to improve longevity. This first generation of the full experiment will be ready to take physics data when CEBAF begins operation again in 2014.

\section{References}

[1] O. Adriani et al. [PAMELA Collaboration], Nature 458, 607 (2009)

[2] M. Ackermann et al. [Fermi LAT Collaboration], Phys. Rev. Lett. 108, 011103 (2012)

[3] B. Holdom, Phys. Lett. B 166, 196 (1986).

[4] N. Arkani-Hamed, D. P. Finkbeiner, T. R. Slatyer and N. Weiner, Phys. Rev. D 79, 015014 (2009)

[5] D. Tucker-Smith and N. Weiner, Phys. Rev. D 64, 043502 (2001)

[6] O. Adriani et al. [PAMELA Collaboration], Phys. Rev. Lett. 105, 121101 (2010)

[7] J. D. Bjorken, R. Essig, P. Schuster and N. Toro, Phys. Rev. D 80, 075018 (2009)

[8] A. Grillo et al., "HPS Heavy Photon Search", Dec. 1, 2010, https://confluence.slac.stanford.edu/download/attachments/86676777/HPSProposal-FINAL_Rev2.pdf

[9] I. Rashevskaya et al., Nucl. Instrum. Meth. A 485, 126 (2002).

[10] D. S. Denisov and S. Soldner-Rembold, FERMILAB-PROPOSAL-0925.

[11] M. J. French et al., Nucl. Instrum. Meth. A 466, 359 (2001).

[12] M. Friedl, C. Irmler and M. Pernicka, Nucl. Instrum. Meth. A 598, 82 (2009).

[13] N. A. Graf, J. Phys. Conf. Ser. 331, 032012 (2011).

[14] R. S. Larsen, Conf. Proc. C 110904, 1981 (2011). 